\title{
Industria 4.0: el reto para las pymes manufactureras de Bogotá, Colombia
}

\author{
Industry 4.0: The Challenge for Manufacturing SMEs in Bogotá, Colombia
}

Citation: Ladino-Fernández, J. M., Briceño-Barrero, D. L. y Rodríguez, L. A. (2022). Industria 4.0: el reto para las pymes manufactureras de Bogotá. Mutis, 12(1). https://doi.org/10.21789/22561498.1784

Recibido: 27 de julio de 2021

Aceptado: 20 de septiembre de 2021

Copyright: (c) 2021 por los autores. Licenciado para Mutis. Este artículo es un artículo de acceso abierto distribuido bajo los términos y condiciones de la licencia Creative Commons Attribution (https:// https://creativecommons.org/licenses/bync-sa/4.0/).

\section{RESUMEN}

La cuarta revolución industrial es una etapa caracterizada por el uso de diferentes tecnologías que implica cambios en la manera como trabajan las empresas. En tanto que la emergencia sanitaria ocasionada por la pandemia de COVID-19 determinó el cierre de un importante número de las empresas manufactureras, la industria 4.0 representa una oportunidad para enfrentar la crisis por parte de las pymes bogotanas del sector manufacturero. La finalidad del presente documento es presentar un diagnóstico relacionado con la implementación de la cuarta revolución industrial en las pequeñas y medianas empresas manufactureras, contribuyendo de esta manera a establecer una hoja de ruta que permita poner en marcha estas tecnologías en las empresas colombianas. La metodología se dividió en tres fases. En la primera se identificaron características de la problemática. La segunda fase consistió en recolectar información por medio de una encuesta a 100 pymes del sector manufacturero. En la tercera fase se documentaron los resultados obtenidos. Dentro de las conclusiones del estudio, se destaca que el $85 \%$ de las empresas no realiza capacitaciones en herramientas de la industria 4.0 y solo $18,6 \%$ de ellas tiene un plan de inversión en esta dimensión; apenas un 3,9\% utiliza software especializado para el tratamiento de sus datos y el $36,3 \%$ de las empresas no usa ningún método de ciberseguridad.

Palabras clave: industria 4.0, pymes, manufactura, competitividad, covid-19

\section{ABSTRACT}

The fourth industrial revolution is a stage characterized by the use of different technologies in all areas, and this means changes in the way companies work. The health emergency caused by the COVID-19 pandemic resulted in the closure of a significant number of manufacturing companies. Industry 4.0 represents an opportunity to face this crisis for SMEs in the manufacturing sector in the city of Bogotá (Colombia). The purpose of this paper is to present a diagnosis related to the implementation of the fourth industrial revolution in small and medium-sized manufacturing companies. In this way, we contribute to establish a roadmap that allows the implementation of these technologies in Colombian companies. The methodology was divided into three phases: in the first, characteristics of the problem were identified; the second phase consisted of collecting information through a survey of 100 SMEs in the manufacturing sector; while the third phase reports the results obtained. Among the conclusions of the study, it is highlighted that $85 \%$ of the companies studied do not carry out training in industry 4.0 tools. Besides, only $18.6 \%$ of these firms 
have an investment plan in 4.0 technologies, just $3.9 \%$ use specialized software to process their data, and $36.3 \%$ of companies do not use any cybersecurity method.

Keywords: Industry 4.0, SMES, manufacturing, competitiveness, covID-19.

\section{INTRODUCCIÓN}

Se denominan revoluciones industriales a los hitos que han marcado las etapas en el camino del desarrollo industrial, como se muestra en la figura 1 . En principio, el concepto de la industria 4.0 surge en Alemania en 2011 . No obstante, es hasta la edición de 2013 de la feria de Hannover donde formalmente se expone esta como la nueva forma de industrialización, mediante la implementación del Internet of things (IOT) en el entorno de fabricación (Feng et al., 2018; Kagermann et al., 2013). El Instituto Alemán de Normalización Industrial define la cuarta revolución industrial como una fusión de los sistemas de producción con el mundo virtual. Es así como la manufactura y la logística se encuentran en constante comunicación por medio de las diferentes herramientas tecnológicas (Kopp \& Basl, 2017).

Figura 1. Evolución de la industria

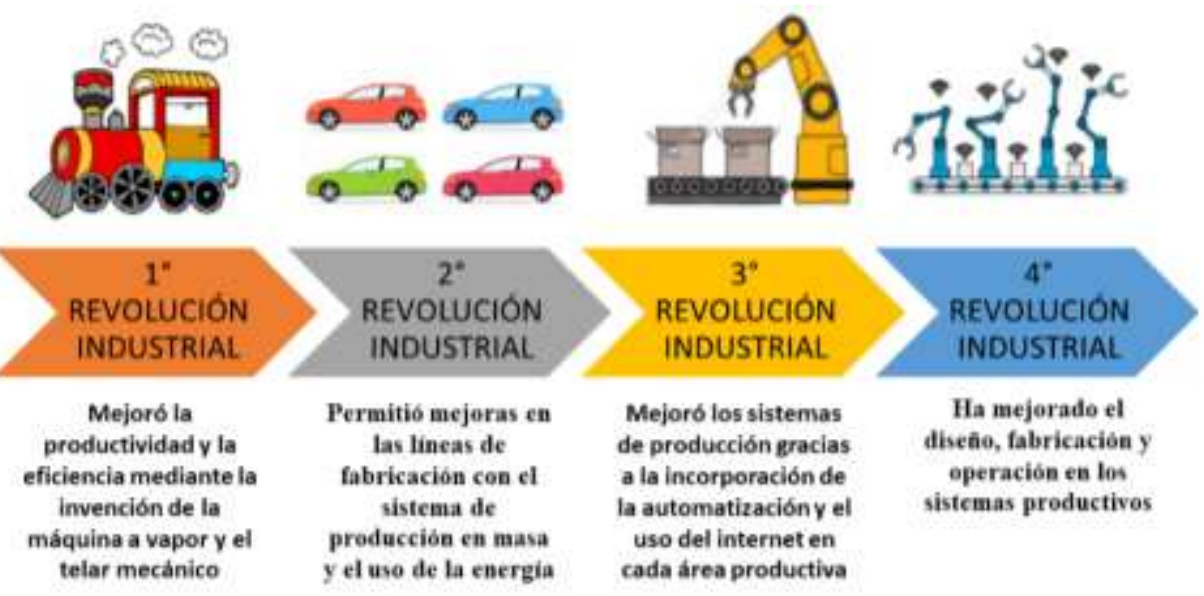

Fuente: elaboración propia.

Cabe mencionar que varios autores concuerdan en definirla como un conjunto de desarrollos tecnológicos que incluyen sistemas ciber-físicos (CPS), internet de las cosas (IoT), internet de servicios (IoS), robótica, big data, fabricación en la nube, realidad aumentada, fabricación aditiva, impresión 3D e inteligencia artificial (Fundación Conama, 2018; Gökalp et al., 2017; Jodlbauer \& Schagerl, 2016). Estas tecnologías implican cambios significativos en la manera como trabajan las empresas. Para ilustrar mejor, en la industria 4.0 los objetos se tornan inteligentes y se utilizan códigos de barras o chips que contienen información relevante para garantizar trazabilidad, así como escáneres o sensores que aportan información y se comunican mediante loT (Sommer, 2015).

Con la incorporación de la industria 4.0 se minimizan los ciclos de producción, las necesidades de los clientes se procesan en tiempo real y el mantenimiento se automatiza en gran medida, dando como resultado las conocidas fábricas inteligentes (Kopp \& Basl, 2017). Por consiguiente, la implementación de estas tecnolo- 
gías permite encontrar soluciones capaces de sobrellevar la creciente complejidad del entorno y garantizar una competitividad sostenible (De-Carolis et al., 2017). Sin embargo, al ser un escenario relativamente nuevo, se debe analizar y preparar una hoja de ruta para implementar dichas tecnologías en las organizaciones, con el fin de romper los viejos paradigmas que caracterizan a las pymes (Jacquez-Hernández \& López Torre, 2018).

Las pymes de son de vital importancia para el desarrollo del país debido a su potencial como generadoras de empleo y crecimiento económico (Schwab, 2018; Ñungo-Pinzón et al., 2018). Actualmente, "Colombia posee 2.540.953 Mipymes, que representan el $90 \%$ de las empresas del país, producen solo el $30 \%$ del PIB y emplean más del $65 \%$ de la fuerza laboral nacional" (Fintech, 2021). Por tal razón, las empresas del sector necesitan del apoyo por parte del Gobierno para encaminarse hacia la industria 4.0 (Vicepresidencia de Transformación Digital, 2019), lo cual constituye un reto, si se tiene en cuenta que la digitalización de los procesos productivos se encuentra muy rezagada. Según la Comisión Económica para América Latina y el Caribe (CEPAL), en las empresas latinoamericanas el uso de tecnologías digitales en la cadena de abastecimiento es solo del 37 \% en países como Brasil, Chile y Colombia (CEPAL, 2020a).

\section{Antecedentes}

La competitividad es definida por el Foro Económico Mundial como "el conjunto de instituciones, políticas y factores que determinan el nivel de productividad de un país" (Schwab, 2019, p. 15). También es entendida como la manera en que las empresas miden su capacidad de competir en el mercado global, logrando conseguir beneficios económicos y expandirse (Fuentes-Pilaló \& Véliz-Salazar, 2018).

Para medir la competitividad de los países existen diferentes herramientas. El desempeño de Colombia evidencia pequeños avances a pesar de los grandes esfuerzos en los últimos años. La tabla 1 muestra el lugar ocupado por el país en la medición más reciente de los últimos índices.

Tabla 1. Índices de competitividad

\begin{tabular}{|c|c|c|}
\hline Índice & $\begin{array}{l}\text { Puesto } \\
\text { Colombia }\end{array}$ & Diferencias/comparación \\
\hline $\begin{array}{l}\text { Índice Global de Competitividad (IGC) del } \\
\text { Foro Económico Mundial }\end{array}$ & $57 / 141$ & $\begin{array}{l}\text { Mide la capacidad que tiene un país de generar oportunidades de desarro- } \\
\text { llo económico para los ciudadanos (Schwab, 2019). }\end{array}$ \\
\hline Índice Global de Innovación (G॥) & $68 / 131$ & $\begin{array}{l}\text { Permite determinar las capacidades y los resultados en materia de inno- } \\
\text { vación de las economías del mundo (WIPO, 2020). }\end{array}$ \\
\hline $\begin{array}{l}\text { IMD Competitividad digital. } \\
\text { The IMD World Digital Competitiveness } \\
\text { Ranking } 2020 \text { results }\end{array}$ & $61 / 63$ & $\begin{array}{c}\text { Mide la capacidad y disposición para adoptar y explorar tecnologías digita- } \\
\text { les como un motor clave para la transformación económica } \\
\text { (Digital Quality of Life Index 2020, 2014). }\end{array}$ \\
\hline Índice de calidad de vida digital Sufshark & $62 / 85$ & $\begin{array}{c}\text { Revisa cinco pilares fundamentales: accesibilidad de internet, calidad de } \\
\text { internet, infraestructura electrónica, seguridad y gobierno electrónicos } \\
\text { (Digital Quality of Life Index 2020, 2014). }\end{array}$ \\
\hline
\end{tabular}

Fuente: elaboración propia. 
Los informes mencionan como causa de estos resultados la falta de estructura digital y acceso al internet, así como también un evidente estancamiento en varios otros aspectos, incluidos la disponibilidad de talento, la efectividad de programas de formación y del sistema educativo y la eficiencia del marco regulatorio. Por el contrario, los gobiernos de los países desarrollados muestran un gran interés por utilizar las tecnologías de la industria 4.0 con el fin de ser más competitivos (Blandon-Andrade, 2020). Así por ejemplo, Emiratos Árabes se convirtió en el primer país en designar un ministro de inteligencia artificial, mientras que aproximadamente veinticinco países han creado estrategias enfocadas en la inteligencia artificial y varios más están desarrollando mecanismos para adoptar loT y blockchain (Bandura et al., 2019).

\section{Situación de las pymes}

La nueva emergencia sanitaria ocasionó que la mayoría de las empresas manufactureras tuviera que detener sus actividades o cerrar, pues no se clasificaban como actividades esenciales (Pérez, 2020). De acuerdo con la Asociación Nacional de Instituciones Financieras (ANIF), para el primer trimestre de 2020 las pequeñas y medianas empresas en los sectores de industria, comercio y servicios presentaron un fuerte deterioro en su situación económica general y una baja en el volumen de ventas, la cual parece no mejorar (ANIF, 2020).

Uno de los sectores más afectados fue el manufacturero, el cual genera un porcentaje de empleo significativo en las ciudades colombianas. En la Cámara de Comercio de Bogotá hay registradas 80.724 empresas del sector, las cuales tienen entre 3 y 15 trabajadores en promedio (Vicepresidencia de Transformación Digital, 2019) y se encuentran clasificadas en subsectores para cada actividad productiva de la siguiente forma: 1 . Alimentos y bebidas; 2 . Algodón, fibras, textiles cuero, calzado y marroquinería; 3 . Madera y muebles; 4 . Papel, cartón y actividades de edición; 5. Refinación de petróleo, químicos y productos de caucho y plástico; 6 . Minerales no metálicos; 7. Metalmecánica y maquinaria; 8. Otros rubros (Rebolledo et al., 2013).

Es así como la CEPAL estima que $34,2 \%$ del empleo formal y $24,6 \%$ del PIB de la región corresponden a sectores fuertemente afectados por la crisis derivada de la pandemia. En Colombia, la caída fue de un 7,7\% para el total de la industria, obteniéndose los peores resultados en los sectores de cuero y calzado $(-37,8 \%)$ y en el de autos y autopartes $(-36,9 \%)$, a diferencia de la industria de alimentos, que registró un crecimiento de 6,1 \% (CEPAL, 2020b).

Además, la industria bogotana se enfrenta a otros desafíos diferentes a los que trajo consigo el coviD-19. Los recientes cambios sociales y tecnológicos, la disminución de la disponibilidad de recursos naturales, los cambios en la demanda, el aumento de los precios de la energía y el fenómeno de la globalización (Erol et al., 2016; Gracel \& Łebkowski, 2019; Jæger \& Halse, 2017; Ganzarain \& Errasti, 2008; Pérez-Lara et al., 2017) hacen que la situación de las pymes de la región sea más complicada. Es por esto que se deben centrar los esfuerzos en diferentes estrategias que permitan al país tener un mejor desempeño competitivo en los mercados, tanto nacionales como externos (Escandón \& Hurtado, 2014). Con relación a las problemáticas expuestas, de acuerdo con datos obtenidos de la Cámara de Comercio de Bogotá (CCB, 2021), el panorama de las mipymes de Bogotá se resume en la figura 2 . 
Figura 2. Reactivación productiva mipymes Bogotá - Región (enero de 2021)

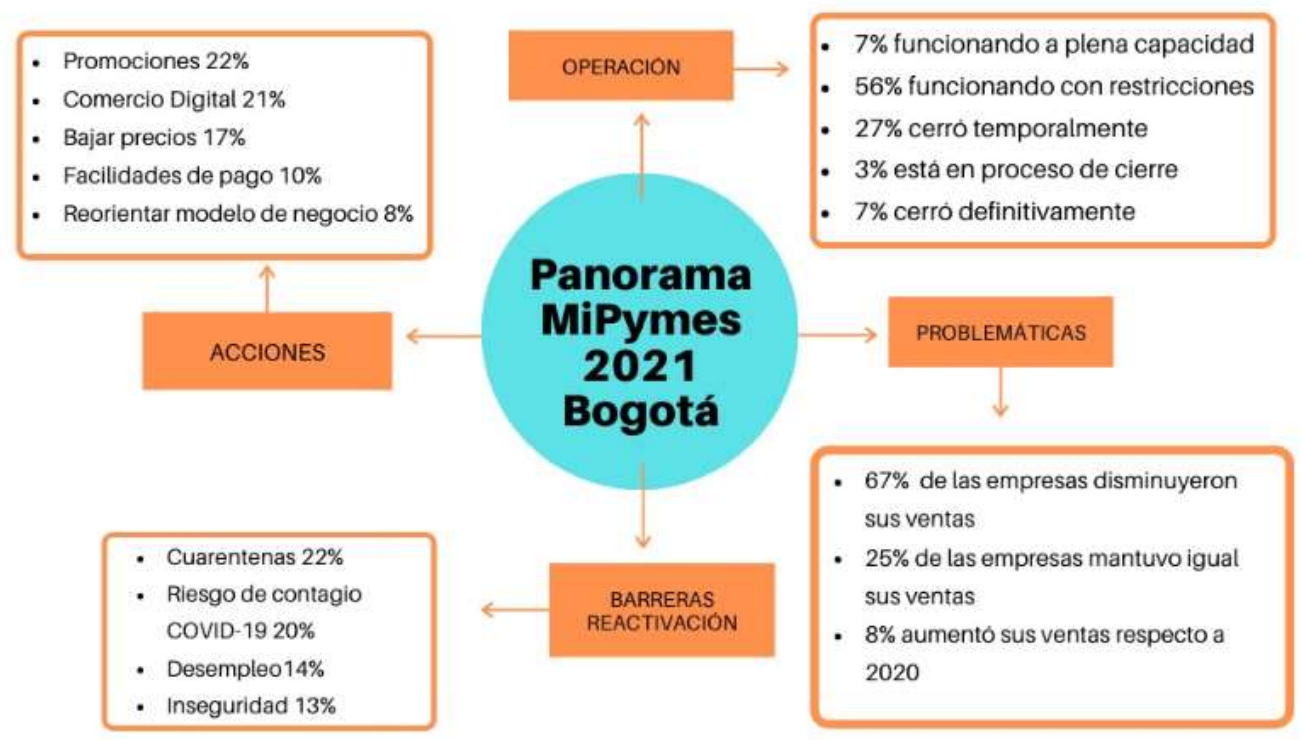

Fuente: adaptado de ссв (2021).

Por lo que se refiere a industria 4.0, para 2019 la gran mayoría de los empresarios conocían las tecnologías emergentes, pero no hacían uso de las mismas (Vicepresidencia de Transformación Digital, 2019). No obstante, se pudo identificar que la tecnología de mayor apropiación en la industria manufactura nacional es el cloud computing, ya que el $71,8 \%$ de los empresarios la usan. Además, el 30,2 \% de las empresas requieren capacitaciones en ventas digitales y atención al cliente a través de plataformas web. El 21,5\% de los empresarios demandan capacitaciones en marketing digital y social media, y un $12 \%$ en manejo de TIC (Confecámaras, 2020).

En este contexto, las tecnologías asociadas a la industria 4.0 representan una oportunidad para fortalecer la capacidad de las empresas y de esta manera reducir el impacto económico que tienen las medidas de aislamiento obligatorio (Confecámaras, 2021). De hecho, la actual crisis sanitaria ha mostrado la posibilidad de implementar dichas tecnologías en el mundo laboral en trabajos que en años anteriores eran impensables (Barrientos-Avendaño et al., 2020).

Dentro de las posibilidades que se contemplan para el desarrollo de las pymes en pospandemia se encuentra dar acceso a crédito para desarrollar planes de inversión encaminados a la recuperación productiva, financiar proyectos que conduzcan a la innovación (Avendaño \& William, 2012), brindar herramientas tecnológicas para facilitar el e-commerce y crear centros que brinden asistencia técnica individual para apoyar en la implementación de tecnologías en los procesos de las pymes. Todo esto se proyecta con el fin de que el sector que más impacta la productividad logre ser autosuficiente y aporte a la reactivación económica del país (Zurita-Heredia \& Dini, 2021). 


\section{Marco legal}

Para estudiar e implementar la industria 4.0 en las pymes bogotanas, es importante mencionar los aspectos legales que rigen el funcionamiento de estas. En Colombia se cuenta con legislación que regula el desarrollo de las mipymes del país, y se evidencia que un alto porcentaje de inversión proviene principalmente de los recursos propios de las organizaciones, pues muchas empresas utilizan la modalidad de microcrédito con capitales de libre inversión (Lis-Gutiérrez et al., 2017).

El Consejo Nacional de Política Económica y Social (Conpes) es la máxima autoridad nacional de planeación y se desempeña como organismo asesor del Gobierno en todos los aspectos relacionados con el desarrollo económico y social del país. En este consejo se estudian y aprueban documentos sobre el desarrollo de políticas generales. Claro ejemplo es el Conpes 3620 del 2010, que estableció los lineamientos para el desarrollo e impulso del comercio electrónico en Colombia, el Conpes 3866 de 2016, el cual definió la Política Nacional de Desarrollo Productivo, y el Conpes 3920, que pretende aumentar el aprovechamiento de datos de las empresas. En la figura 3 se mencionan los documentos Conpes más importantes de los últimos años.

Figura 3. Marco legal colombiano para las pymes

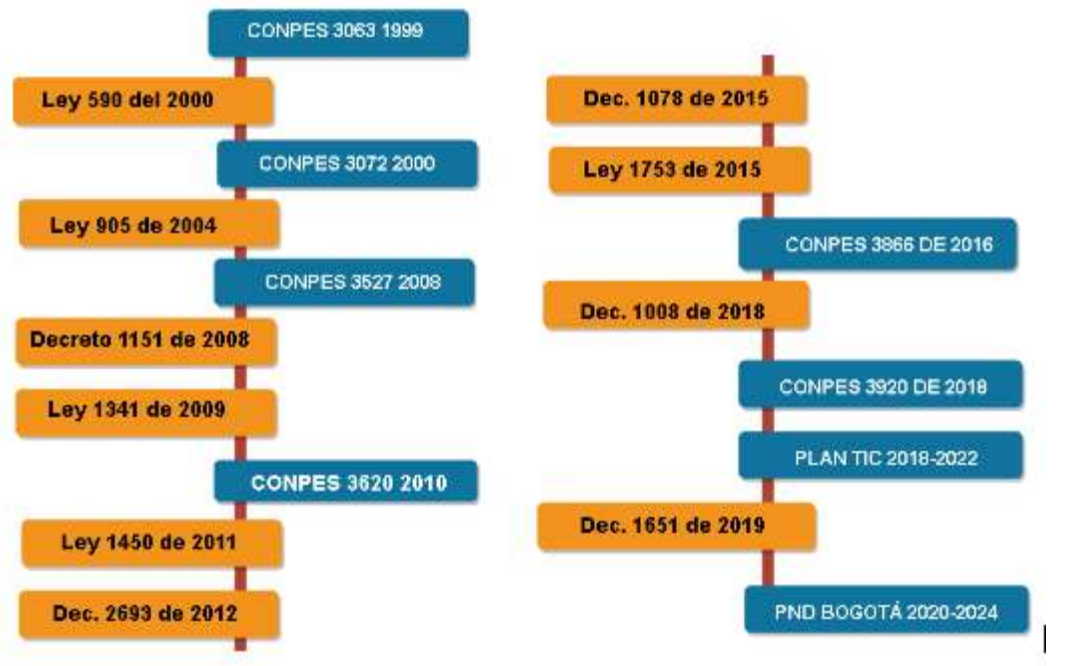

Fuente: elaboración propia.

En respuesta a la necesidad de las pymes de implementar nuevas tecnologías en sus operaciones, el actual Plan Nacional de Desarrollo (2018-2022) constituye un importante avance, pues trabaja temas como emprendimiento, transformación digital, ciencia, tecnología e innovación (Vallejo-Zamudio, 2019). La meta es que las empresas colombianas diversifiquen su producción, generen un mayor valor agregado y aumenten las exportaciones usando las nuevas tecnologías (Confecámaras, 2021).

Así mismo, en 2006 se creó el Consejo Privado de Competitividad (CPC), un grupo de empresarios comprometidos con el desarrollo de Colombia, cuyo objetivo es lograr que a 2030 el país se encuentre entre los tres más competitivos de América Latina. Desde su creación, el CPC ha logrado incidir de manera efectiva en política pública, en temas tales como: costos de transporte y facilitación de comercio, sis- 
tema tributario más competitivo, descuento por inversión en bienes de capital y sostenibilidad ambiental (CPC, 2019).

\section{METOdOLOGíA}

El desarrollo de este trabajo se dividió en tres fases. La primera consistió en realizar un diagnóstico de las pymes del sector manufacturero en el país y su nivel de preparación hacia la industria 4.0; la segunda fase en recolectar información mediante una encuesta a una muestra de pymes del sector manufacturero; la tercera implicó documentar los resultados obtenidos.

Para realizar el primer acercamiento a la problemática se realizó búsqueda de estudios o investigaciones con los términos "Industry 4.0", "SMES", "manufacture" y "competitiveness" en bases de datos como Science Direct, Springer, Google Scholar y Scopus, obteniendo los resultados que se detallan en la tabla 2.

Tabla 2. Resultados de la búsqueda en bases de datos

\begin{tabular}{|c|c|c|}
\hline Fuente & Registros encontrados & Registros relevantes \\
\hline Science Direct & 95 & 24 \\
\hline Springer & 121 & 84 \\
\hline Google Scholar & 782 & 42 \\
\hline Scopus & 181 & 69 \\
\hline
\end{tabular}

Fuente: elaboración propia.

De los artículos revisados se seleccionaron aquellos que contenían en su título al menos tres de las palabras clave, reduciendo el número a ochenta publicaciones. Adicional a las bases de datos científicas, se consultaron informes publicados por entidades como el Departamento Nacional de Planeación (DNP), la Asociación Nacional de Empresarios de Colombia (ANDI), Confecámaras y la CEPAL.

A partir de las publicaciones seleccionadas se realizó una revisión sistemática, la cual permitió identificar los principales aspectos a tener en cuenta para implementar tecnologías de la cuarta revolución industrial en las organizaciones a nivel mundial. En la tabla 3 se presentan las variables claves para conocer la preparación de las pymes en materia de industria 4.0.

Tabla 3. Variables clave para la competitividad de las pymes

\begin{tabular}{|c|c|c|c|}
\hline Dimensión & Variable & Descripción de la variable & Fuente \\
\hline \multirow{2}{*}{$\begin{array}{l}\text { Gestión del } \\
\text { talento humano }\end{array}$} & $\begin{array}{l}\text { Nivel de } \\
\text { formación }\end{array}$ & $\begin{array}{l}\text { Conocimiento, competencias y habilida- } \\
\text { des del personal en cuanto a industria } \\
\text { 4.0. }\end{array}$ & $\begin{array}{l}\text { Rojko (2017), Schuh et al. (2020), Vivares- } \\
\text { Vergara (2017), Schumacher et al. (2016), } \\
\text { Restrepo-Morales y Vanegas López (2015) }\end{array}$ \\
\hline & Capacitación & $\begin{array}{l}\text { La empresa brinda capacitación a sus } \\
\text { trabajadores en cuanto a tecnologías de } \\
\text { la industria } 4.0 \text {. }\end{array}$ & $\begin{array}{c}\text { Greif et al. (2016), Restrepo-Morales y } \\
\text { Vanegas-López (2015) }\end{array}$ \\
\hline \multirow[t]{2}{*}{ Estrategia } & $\begin{array}{l}\text { Gestión de cono- } \\
\text { cimiento }\end{array}$ & $\begin{array}{l}\text { Acceso, análisis e intercambio de cono- } \\
\text { cimientos por parte de todos los traba- } \\
\text { jadores. }\end{array}$ & Gracel y Łebkowski, (2019), Zorro-Galindo (2019) \\
\hline & Mantenimiento & $\begin{array}{l}\text { Cómo monitorea el estado de la maqui- } \\
\text { naria. }\end{array}$ & $\begin{array}{c}\text { Schuh et al. (2020), Vivares-Vergara (2017), } \\
\text { De-Carolis et al. (2017) }\end{array}$ \\
\hline
\end{tabular}


Ladino-Femándlez, J . M., Briceño-Barrero, D. L y Rodríguez, L A. (2022). https://doi.org/10.21789/22561498.1784

\begin{tabular}{|c|c|c|c|}
\hline & Oferta al cliente & $\begin{array}{l}\text { Medios por los cuales se ofrece el pro- } \\
\text { ducto al cliente. }\end{array}$ & $\begin{array}{l}\text { Schumacher et al. (2016), Klötzer y Pflaum } \\
\text { (2017), Estrella-Ramón et al. (2012) }\end{array}$ \\
\hline & Plan de inversión & $\begin{array}{l}\text { La empresa tiene un plan de inversión } \\
\text { destinado a tecnologías de la industria } \\
\text { 4.0. }\end{array}$ & $\begin{array}{l}\text { Gracel y Łebkowski (2019), } \\
\text { Vivares-Vergara (2017), Soldatos et al. (2016) }\end{array}$ \\
\hline \multirow{2}{*}{ Recursos } & Inversión & $\begin{array}{l}\text { Cantidad invertida en implementar las } \\
\text { tecnologías } 4.0 \text {. }\end{array}$ & $\begin{array}{l}\text { De-Carolis et al. (2017), Gökalp et al. (2017), } \\
\text { Gracel y Łebkowski (2019), } \\
\text { Pérez-Lara et al. (2017), Schumacher et al. (2016) }\end{array}$ \\
\hline & Tecnología & $\begin{array}{l}\text { Tipos de tecnología de la industria } 4.0 \\
\text { con las que cuenta la empresa. }\end{array}$ & Gracel y Łebkowski (2019), Zorro-Galindo (2019) \\
\hline \multirow{4}{*}{$\begin{array}{l}\text { Gestión de } \\
\text { datos }\end{array}$} & $\begin{array}{l}\text { Recolección de la } \\
\text { información }\end{array}$ & $\begin{array}{l}\text { Medios utilizados para la recopilación de } \\
\text { información. }\end{array}$ & De-Carolis et al. (2017) \\
\hline & $\begin{array}{l}\text { Análisis de la } \\
\text { información }\end{array}$ & $\begin{array}{l}\text { Herramientas utilizadas para el trata- } \\
\text { miento de datos. }\end{array}$ & Gökalp et al. (2017), Jodlbauer y Schagerl (2016) \\
\hline & $\begin{array}{l}\text { Integración de la } \\
\text { información }\end{array}$ & $\begin{array}{l}\text { Métodos o herramientas utilizadas para } \\
\text { la distribución de información dentro de } \\
\text { la organización. }\end{array}$ & $\begin{array}{l}\text { Gökalp et al. (2017), } \\
\text { Restrepo-Morales y Vanegas-López (2015) }\end{array}$ \\
\hline & $\begin{array}{l}\text { Trazabilidad del } \\
\text { producto }\end{array}$ & $\begin{array}{l}\text { Capacidad para dar seguimiento a los } \\
\text { productos/servicio. }\end{array}$ & Vivares-Vergara (2017) \\
\hline Ciberseguridad & $\begin{array}{l}\text { Ciberseguridad } \\
\text { (en recursos) }\end{array}$ & $\begin{array}{l}\text { Medios o herramientas para prevenir y } \\
\text { procesar los riesgos de ciberseguridad. }\end{array}$ & $\begin{array}{l}\text { De-Carolis et al. (2017), Gracel y } \\
\text { Łebkowski (2019), Rojko (2017) }\end{array}$ \\
\hline
\end{tabular}

Fuente: elaboración propia.

Para la segunda fase de la investigación se diseñó una encuesta con veinte preguntas, que fue aplicada mediante formulario virtual entre abril y mayo de 2021 a una muestra de empresas del sector manufacturero, determinada mediante un muestreo no probabilístico de tipo convencional (Otzen \& Manterola, 2017), de la cual se obtuvieron 100 respuestas. Desde el punto de vista del teorema del límite central, al seleccionar muestras aleatorias simples de tamaño $n$ de una población, la distribución de la media muestral $X$ se puede aproximar a una función de probabilidad normal, cuando el tamaño de la muestra es grande; es debido a ello que una muestra de 100 empresas es suficiente para que se emplee el teorema del límite central (Vargas, 2008).

\section{RESULTADOS}

La caracterización de las empresas muestra los resultados presentados en la figura 4. A partir de la información recolectada de las empresas encuestadas, los canales que estas utilizan para ofrecer sus productos al cliente están representados en un $77 \%$ por medios físicos, $65 \%$ por redes sociales y $29 \%$ por medio de páginas web. Así mismo, la recopilación de datos e información se realiza en un 49 \% por medio de redes sociales, y un 35,3\% de manera presencial o telefónica, mientras que el 29,4\% de las empresas no recopila datos o información. 
Figura 4. Actividad y cantidad de empleados de las empresas encuestadas
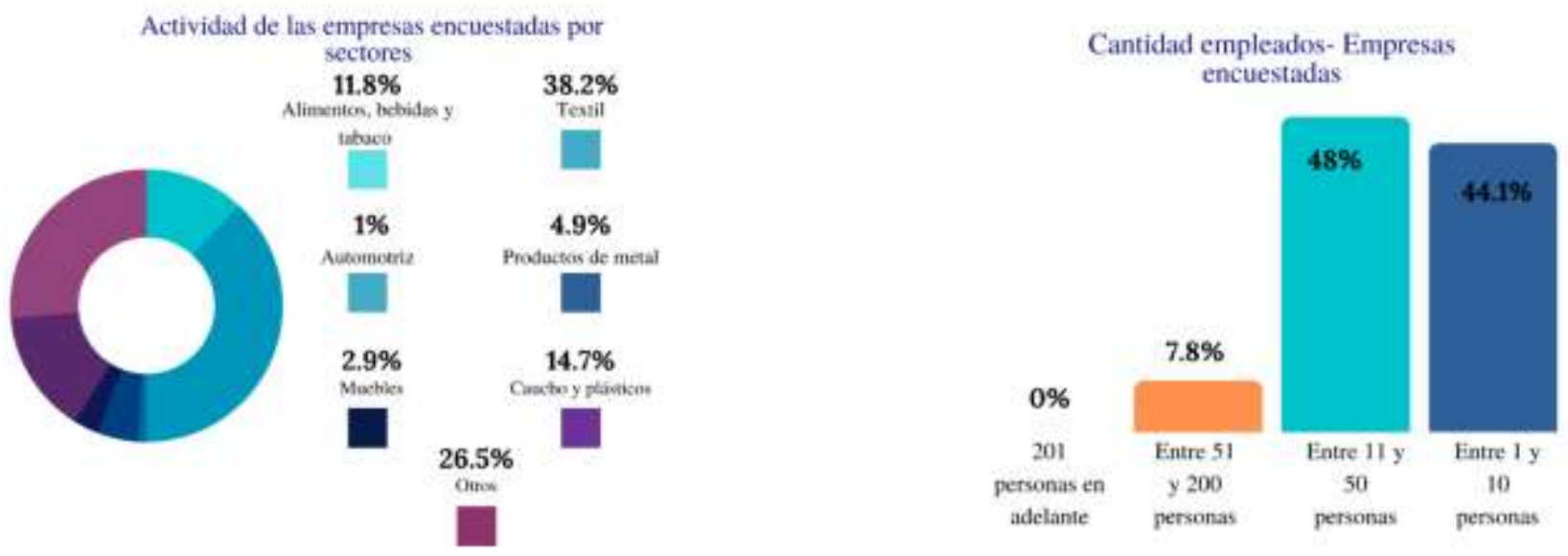

Fuente: elaboración propia.

El método más utilizado para el intercambio de información de las pymes bogotanas es el de las reuniones presenciales $(85,3 \%)$, seguido de las reuniones virtuales $(41,2 \%)$, el correo electrónico $(40,2 \%)$ y los reportes escritos $(13,7 \%)$. Cabe destacar que las empresas resaltan el papel del móvil como herramienta para el intercambio de información. Por otra parte, $6 \%$ de las empresas encuestadas manifiesta no intercambiar información.

En cuanto al tratamiento de datos, $52,9 \%$ de las empresas realiza esta actividad mediante ofimática, $36,3 \%$ no analiza sus datos, 6,9 \% lo realiza en la nube y $3,9 \%$ lo hace por medio de software especializado. Finalmente, dentro de las herramientas que se utilizan para prevenir los riesgos de ciberseguridad, el 60,8\% respondió que usa antivirus, $24,5 \%$ usa cortafuegos (firewall), 17,6\% filtros web y $36,3 \%$ no usa ningún método de ciberseguridad.

En cuanto al nivel de conocimientos, habilidades y aptitudes de los empleados, el $83,7 \%$ de las empresas reporta que menos del $25 \%$ de sus empleados se encuentra familiarizado con tecnologías de la cuarta revolución industrial. 
Figura 5. Conocimientos y aptitudes de los trabajadores en las pymes de manufactura de Bogotá-región en cuanto a industria 4.0

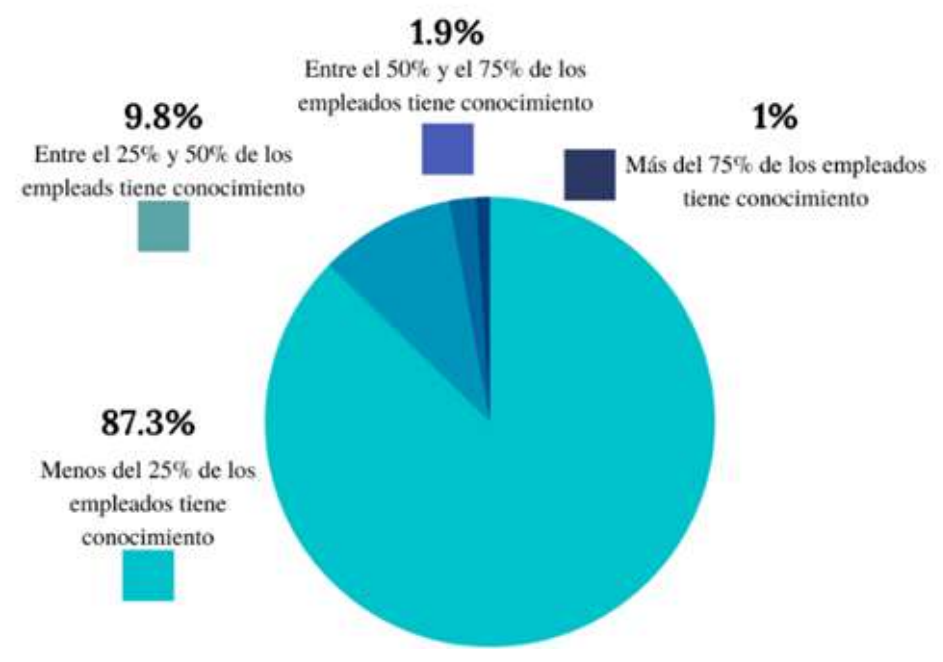

Fuente: elaboración propia.

Las capacitaciones que se realizan en las pymes están enfocadas en loT en un $50 \%$, seguido de big data y computación en la nube (43\%), blockchain, robótica y manufactura aditiva (12,5\%) y, finalmente, inteligencia artificial (6,3\%). Las áreas donde se aplican las capacitaciones relacionadas con la industria 4.0 corresponden en $56,1 \%$ a todas las áreas de las empresas encuestadas, 38,8 \% al área directiva, 37,8 \% al área productiva y 5,1\% a áreas de apoyo. Sin embargo, el 85,3\% de las empresas no realiza ningún tipo de capacitación relacionada con herramientas de la industria 4.0 (figura 6).

Figura 6. Capacitaciones realizadas en las pymes de manufactura de Bogotá-región en cuanto a industria 4.0

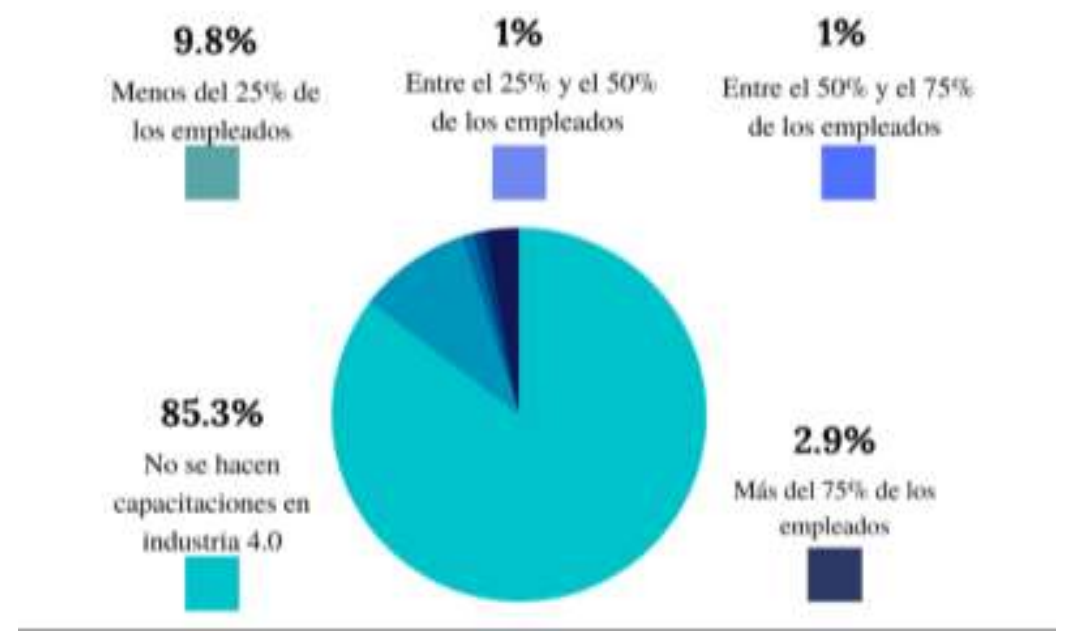

Fuente: elaboración propia. 
Dentro de las barreras para implementar las tecnologías de la industria 4.0, los empresarios destacan las limitaciones señaladas en la figura 7.

Figura 7. Principales barreras en pymes de manufactura de Bogotá-región en cuanto a industria 4.0

\section{$71.6 \%$}

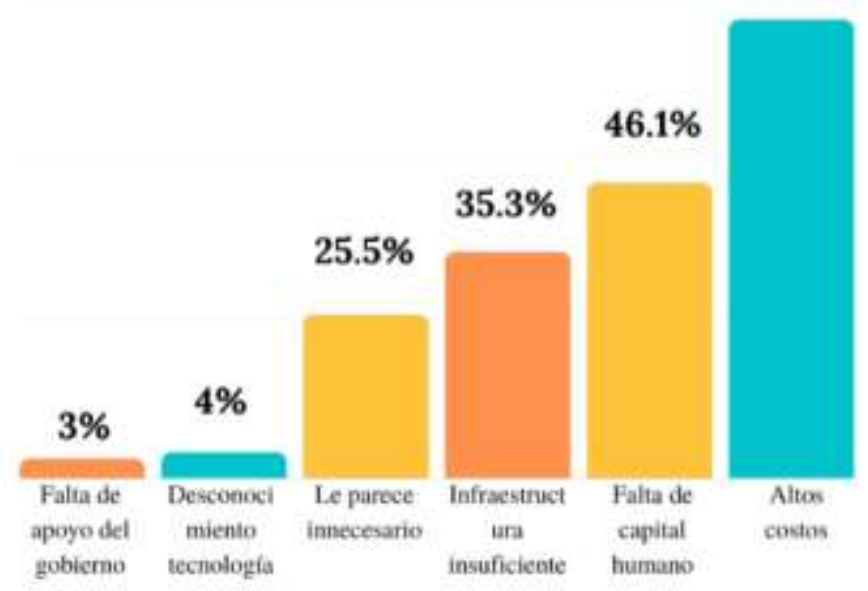

Fuente: elaboración propia.

Desde el punto de vista financiero, tan solo $18,6 \%$ de las empresas tiene un plan de inversión en tecnología 4.0 y menos del $1 \%$ del presupuesto anual de las empresas se destina a la industria 4.0 en el 68 \% de los casos. Así mismo, las tecnologías utilizadas por las pymes de la región hasta el momento se distribuyen como lo muestra figura 8.

Figura 8. Uso de tecnologías propias de la industria 4.0 en las pymes de manufactura de Bogotá-región

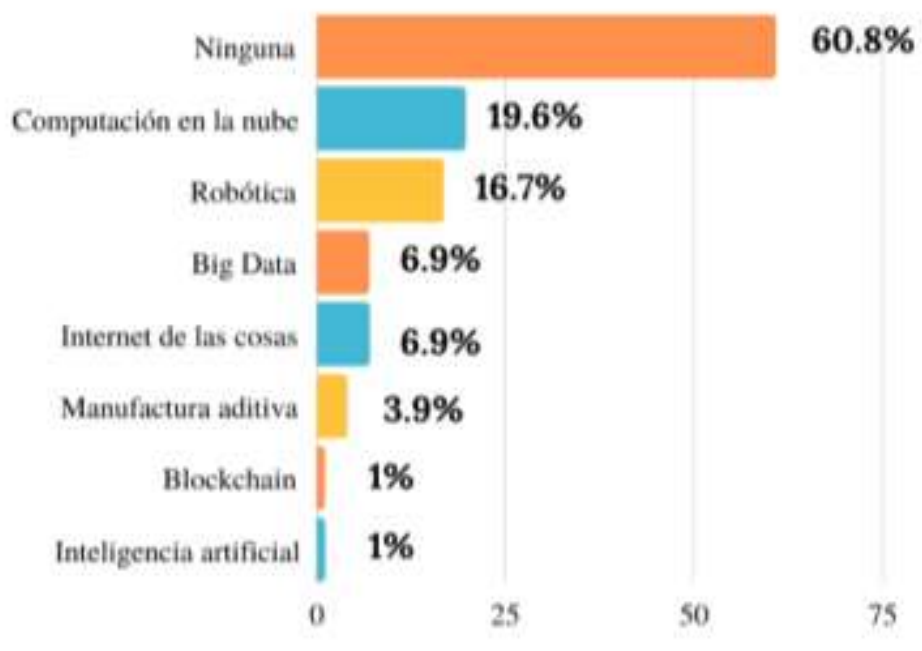

Fuente: elaboración propia. 


\section{DISCUSIÓN}

Los resultados muestran un bajo nivel de adopción de tecnologías de la industria 4.0 por parte de las pymes manufactureras de Bogotá, lo cual puede deberse a que muchas empresas de producción ven esta cuarta revolución como algo más parecido a una moda que a una alternativa de mejora y eficiencia (Lazarte et al., 2018).

El $65 \%$ de los empresarios ha hecho uso de las redes sociales para promocionar sus productos, lo cual concuerda con la encuesta realizada por Confecámaras (2020), la cual muestra que el $63 \%$ de los empresarios ha implementado las redes sociales como canal de venta. Este cambio de canales de venta presenciales a canales virtuales es consecuencia de la crisis sanitaria en la industria, por lo que el e-commerce representa así una gran oportunidad para los empresarios (Confecámaras, 2021).

Una de las barreras para la implementación de la industria 4.0 radica en la poca capacitación de los empleados en estas tecnologías, e incluso en su desconocimiento. A pesar de que el $29 \%$ de las industrias bogotanas realizó capacitaciones como acción de mejora a nivel organizacional (ANIF, 2019), lo clave sería orientar tales capacitaciones hacia la industria 4.0, de manera que los empleados se encuentren preparados para ser más productivos.

La principal barrera para acceder a las tecnologías de la industria 4.0 se refiere a sus altos costos y la dificultad de financiamiento, lo cual puede explicarse porque el $70 \%$ de las empresas industriales en Bogotá se financian con recursos propios y no acceden a recursos externos o a endeudamiento. La principal razón por la que no se accede a créditos es porque no se considera necesario (ANIF, 2019), fenómeno indicador de la poca sinergia existente entre el Gobierno, la banca privada y la industria, ya sea por falta de conocimiento de las diferentes líneas de financiación externa o de patrocinio gubernamental, o bien por la poca difusión de dichos programas.

En materia de ciberseguridad, cerca del $43 \%$ de las empresas colombianas no están preparadas para enfrentar los ciberataques (Ospina-Díaz \& Sanabria-Rangel, 2020), consecuentemente con el resultado de las encuestas, que mostró que un $36,3 \%$ de las empresas entrevistadas no contemplan herramientas para prevenir ataques cibernéticos y el $60,8 \%$ solo utiliza antivirus, por lo que no se cuenta con suficiente protección para la información sensible y confidencial de las empresas.

\section{CONCLUSIONES}

Las pymes del sector manufacturero de Bogotá muestran un panorama en donde se destaca que el $85 \%$ de ellas no realiza capacitaciones en herramientas de la industria 4.0. Solo un $18,6 \%$ de estas tiene un plan de inversión en tecnologías 4.0. El $60 \%$ no utiliza ninguna de las herramientas relacionadas con computación en la nube (big data, blockchain, inteligencia artificial, loT, robótica e impresión 3D). El 29,4\% aún no saca provecho de sus datos, porque no los recopilan. Apenas un 3,9\% utiliza software especializado para el tratamiento de sus datos y el $36,3 \%$ no usa ningún método de ciberseguridad. 
Los resultados muestran una gran necesidad de apoyo hacia las empresas más pequeñas, las cuales han sido las más afectadas después de la llegada de la pandemia. La crisis hizo que centraran sus recursos en las actividades fundamentales para su funcionamiento, como el pago de nómina de sus trabajadores, arriendos, servicios públicos, insumos, materia prima, entre otros, dejando de lado temas como la innovación, la digitalización y las tecnologías de la industria 4.0.

Esta tecnología trae grandes desafíos para la industria colombiana, pues a pesar de los esfuerzos, persiste poco acompañamiento y apoyo por parte del Gobierno nacional en ámbitos como capacitación y formación, maquinaria y financiación de innovaciones de la cuarta revolución industrial, por lo cual resulta conveniente que se gestionen políticas públicas, en consenso con los gremios, para lograr una apropiación de la industria 4.0 y fomentar así el desarrollo del país.

Definir una hoja de ruta puede ayudar a visibilizar un plan de acción que permita a las pymes aprovechar los beneficios de la industria 4.0. Implementar estas tecnologías requiere grandes esfuerzos, pero trae consigo beneficios como el empoderamiento de los líderes de las organizaciones, el uso de la información para aumentar la productividad, la mejora de los procesos y la permanencia en el mercado, la reducción de los errores en los procesos productivos, la predicción de los problemas antes de que sucedan, la toma de decisiones basada en evidencia y, lógicamente, el aumento de la satisfacción del cliente mediante productos y servicios a la medida de sus necesidades.

La academia juega un papel fundamental en este tipo de escenarios: por un lado, uno de los principales desafíos es que no se cuenta con capital humano capacitado que apoye los procesos; por el otro lado, son pocos los estudios en el país que permiten conocer la realidad de las empresas en el ámbito del uso de tecnologías 4.0. Programas académicos acordes con el avance de la tecnología y líneas de investigación enfocadas en las principales dificultades encontradas en este estudio pueden promover el cambio hacia el fortalecimiento de las pymes, contribuyendo de paso a la competitividad del país.

\section{REFERENCIAS}

Asociación Nacional de Instituciones Financieras [ANIF]. (2019). Gran encuesta pyme regional. ANIF. https://www.anif.com.co/file-category/gran-encuesta-pymeregional/

Asociación Nacional de Instituciones Financieras [ANIF]. (2020). Gran encuesta pyme. Lectura nacional. ANIF. http://www.anif.com.co/file-category/gran-encuestapyme-nacional/

Avendaño, C., \& William, R. (2012). Innovación: un proceso necesario para las pequeñas y medianas empresas del municipio de San José de Cúcuta, Norte de Santander (Colombia). Semestre Económico, 15(31), 187-207. https://doi.org/10.22395/seec.v15n31a8

Bandura, R., Campbell-Zausner, C., Carter, W., Hammond, M., Jorge, S., Klynge, C., Milner, A., Nakagaki, M., Raymond, P., Rubin, N., Runde, D. F., \& Zausner, S. (2019). Beyond technology. The fourth industrial revolution in the developing world. Center for Strategic and International Studies. https://www.csis.org/analysis/beyond- 
technology-fourth-industrial-revolution-developing-world

Barrientos-Avendaño, E., Areniz-Arevalo, Y., Coronel-Rojas, L. A., CuestaQuintero, F., \& Rico-Bautista, D. (2020). Industry foray model 4.0 applied to the food company your gourmet bread sas: Strategy for rebirth in the covID-19 (SARS-CoV-2) pandemic. RISTI - Revista Iberica de Sistemas e Tecnologias de Informacao, 2020(E34), 436-449. https://www.scopus.com/inward/record.uri?eid=2-s2.085092249702\& partner ID $=40 \& m d 5=01 b 8650 b 8 d 1 f 4 b 7$ ce76a6de617f5b753

Blandon-Andrade, J. (2020). Tendencias en ciencias de la computación. Entre Ciencia e Ingeniería, 14(27), 19-28. https://doi.org/10.31908/19098367.1740

Cámara de Comercio de Bogotá [CCB]. (2021). Encuesta mensual sobre la reactivación productiva: Mipymes Bogotá - Región. Cuarta medición. СCB. https://bibliotecadigital.ccb.org.co/bitstream/handle/11520/26612/Encuesta mensual sobre la reactivación productiva CCB Febrero 2021. pdf?sequence=1\&isAllowed=y

Comisión Económica para América Latina y el Caribe [CEPAL]. (2020a). Universalizar el acceso a las tecnologías digitales para enfrentar los efectos del covID19. ONU-CEPAL. https://repositorio.cepal.org/bitstream/handle/11362/45938/S2000550_es.pdf?sequ ence $=4 \&$ isAllowed $=y$

Comisión Económica para América Latina y el Caribe [CEPAL]. (2020b). Sectores y empresas frente al COVID-19: emergencia y reactivación. ONU-CEPAL. https://repositorio.cepal.org/bitstream/handle/11362/45734/4/S2000438_es.pdf

Confederación Colombiana de Cámaras de Comercio [Confecámaras]. (2020). Encuesta de las Cámaras de Comercio sobre el Impacto de la Covid-19 en las empresas colombianas.

Confecámaras. http://www.confecamaras.org.co/phocadownload/2020/Encuesta Cámaras de Comercio consolidada.pdf

Confederación Colombiana de Cámaras de Comercio [Confecámaras].(2021). Industria 4.0, Transformación empresarial para la reactivación económica. Confecámaras.

https://www.confecamaras.org.co/phocadownload/2020/Analisis_Economicos/Indust ria\%204.0,\%20Transformaci\%C3\%B3n\%20Empresarial\%20para\%20la\%20Reactivaci\%C 3\%B3n\%20Econ\%C3\%B3mica.pdf

Consejo Privado de Competitividad [CPC]. (2019). Informe Nacional de Competitividad 2019-2020. CPC. https://compite.com.co/informe/informe-nacionalde-competitividad-2019-2020/

De-Carolis, A., Macchi, M., Negri, E., \& Terzi, S. (2017). A maturity model for assessing the digital readiness of manufacturing companies. En H. Lödding, R. Riedel, K. D. Thoben, G. von Cieminski, \& D. Kiritsis (eds.), Advances in Production management systems. The path to intelligent, collaborative and sustainable manufacturing (pp. 13-20) [IFIP Advances in Information and Communication Technology, vol. 513]. Springer. https://doi.org/10.1007/978-3-319-66923-6_2 
Digital Quality of Life Index 2020. (2014). En A. C. Michalos (ed.), Encyclopedia of Quality of Life and Well-Being Research. Springer. https://doi.org/10.1007/978-94-007-0753-5_100965

Erol, S., Schumacher, A., \& Sihn, W. (2016). Strategic guidance towards industry $4.0-\mathrm{A}$ three-stage process model. Internantional Conference on Competitive Manufacturing.

https://www.researchgate.net/publication/286937652_Strategic_guidance_towards_ Industry_40_-_a_three-stage_process_model

Escandón, D. M., \& Hurtado, A. (2014). Determinants of export orientation and performance in SMEs exporters in Colombia. Estudios Gerenciales, 30(133), 430440. https://doi.org/10.1016/j.estger.2014.05.002

Estrella-Ramón, A., Jiménez-Castillo, D., Ruiz-Real, J. L., \& Sánchez-Pérez, M. (2012). ¿Cómo compiten las pymes en los mercados internacionales? Análisis de un clúster local con vocación exportadora. Investigaciones Europeas de Dirección y Economía de la Empresa, 18(1), 87-99. https://doi.org/10.1016/S11352523(12)60062-8

Feng, L., Zhang, X., \& Zhou, K. (2018). Current problems in China's manufacturing and countermeasures for industry 4.0. EURASIP Journal on Wireless Communications and Networking, 2018(90). https://doi.org/10.1186/s13638-018$1113-6$

Fintech. (2021). Pymes también deben implementar la nómina electrónica. Colombia Fintech. https://www.colombiafintech.co/novedades/pymes-tambiendeben-implementar-la-nomina-electronica

Fuentes-Pilaló, E., \& Véliz-Salazar, G. (2018). La competitividad global. Empresarial, 11(44), 40-46. https://doi.org/10.23878/empr.v11i44.113

Fundación Conama. (2018). Tecnología e industria 4.0: la sostenibilidad en la cuarta era industrial. Fundación Conama. http://www.conama.org/conama/download/files/conama2018//GTs\%202018/20_fin al.pdf

Ganzarain, J., \& Errasti, N. (2008). Three stage maturity model in SME's towards Industry 4.0. Journal of Industrial Engineering and Management, 9(5), 11191128. http://dx.doi.org/10.3926/jiem.2073

Gökalp, E., Sener, U., \& Eren, E. (2017). Development of an assessment model for Industry 4.0: Industry 4.0-MM. En A. Mas, A. Mesquida, R. V. O'Connor, T. Rout, \& A. Dorling (eds.) Software process improvement and capability determination (pp. 128-142). Springer. https://doi.org/10.1007/978-3-319-67383-7

Gracel, J., \& Łebkowski, P. (2019). The concept of Industry 4.0 related manufacturing technology maturity model (Manutech Maturity Model, MTMM). Decision Making in Manufacturing and Services, 12, 17-31. https://doi.org/10.7494/dmms.2018.12.1-2.17 
Greif, H., Kühnis, N., \& Warnking, P. (2016). Digitalisierung - Wo stehen Schweizer KMU? PwC Schweiz, Google Switzerland GmbH \& digitalswitzerland. https://www.pwc.ch/de/publications/2016/pwc_digitalisierung_wo_stehen_schweize r_kmu.pdf

Jacquez-Hernández, M. V. \& López-Torre, V. G. (2018). Modelos de evaluación de la madurez y preparación hacia la industria 4.0: una revisión de literatura. Ingeniería Industrial. Actualidad y Nuevas Tendencias, 6(20), 61-78. https://www.redalyc.org/jatsRepo/2150/215057003004/215057003004.pdf

Jæger, B., \& Halse, L. L. (2017). The loT technological maturity assessment scorecard: A case study of Norwegian manufacturing companies. En H. Lödding, R. Riedel, K. D. Thoben, G. von Cieminski, \& D. Kiritsis (eds.), Advances in production management systems. The path to intelligent, collaborative and sustainable manufacturing (pp. 143-150) [IFIP Advances in Information and Communication Technology, vol. 513]. Springer. https://doi.org/10.1007/978-3-319-66923-6_17

Jodlbauer, H., \& Schagerl, M. (2016). Reifegradmodell Industrie 4.0 - Ein Vorgehensmodell zur Identifikation von Industrie 4.0 Potentialen. Lecture Notes in Informatics (LNI), Proceedings - Series of the Gesellschaft fur Informatik (GI), P-259, 1473-1487.

https://dl.gi.de/bitstream/handle/20.500.12116/1035/1473.pdf?sequence=1\&isAllow ed $=y$

Kagermann, H., Wahlster, W., \& Helbig, J. (2013). Recommendations for implementing the strategic initiative INDUSTRIE 4.0. Final report of the Industrie 4.0 Working Group. Acatech. https://www.din.de/blob/76902/e8cac883f42bf28536e7e8165993f1fd/recommendat ions-for-implementing-industry-4-0-data.pdf

Klötzer, C., \& Pflaum, A. (2017). Toward the development of a maturity model for digitalization within the manufacturing industry's supply chain. Proceedings of the 50th Hawaii International Conference on System Sciences (2017), 4210-4219. https://doi.org/10.24251/hicss.2017.509

Kopp, J., \& Basl, J. (2017). Study of the readiness of Czech companies to the Industry 4.0. Journal of Systems Integration, 8(3), 39-45. http://sijournal.org/index.php/JSI/article/view/313

Lazarte, J., Chávez, M., \& Alvarado, M. (2018). Estrategias de aplicación de Industria 4.0 en las empresas peruanas. 16th LACCEI International Multi-Conference for Engineering, Education, and Technology: "Innovation in Education and Inclusion" [1921 July 2018, Lima, Perú], 1-9. https://1library.co/document/qo3vj20q-estrategiasaplicacion-industria-empresas-peruanas.html

Lis-Gutiérrez, J. P., Palencia-Fajardo, O., \& Gaitán-Angulo, M. (2017). Caracterización de las pymes de confecciones en Neiva (Colombia). Suma de Negocios, 8(17), 57-62. https://doi.org/10.1016/j.sumneg.2017.03.002 
Ñungo-Pinzón, L. T., Torres-González, B., \& Palacios-Osma, J. I. (2018). Modelo de nivel de madurez para los procesos de emprendimiento en las pymes $\begin{array}{llll}\text { colombianas. Ingeniería } & \text { Solidaria, } & \text { 14(26), }\end{array}$ https://doi.org/10.16925/in.v14i26.2456

Ospina-Díaz, M. R., \& Sanabria-Rangel, P. E. (2020). Desafíos nacionales frente a la ciberseguridad en el escenario global: un análisis para Colombia. Revista Criminalidad, 62(2), 199-217. http://www.scielo.org.co/pdf/crim/v62n2/1794-3108crim-62-02-199.pdf

Otzen, T., \& Manterola, C. (2017). Técnicas de muestreo sobre una población a estudio. International Journal of Morphology, 35(1), 227-232. https://doi.org/10.4067/S0717-95022017000100037

Pérez, E. L. (2020). La industria 4.0 y las nuevas formas de trabajar: una perspectiva desde el caso mexicano en tiempos del covid 19. Lan Harremanak Revista de Relaciones Laborales, 43, 244-263. https://doi.org/10.1387/lanharremanak.21737

Pérez-Lara, M., Saucedo-Martínez, J. A., Salais-Fierro, T. E., \& MarmolejoSaucedo, J. A. (2017). Caracterizacion de modelo de negocio en el marco de industria 4.0. Congreso Internacional de Logística y Cadena de Suministro (Cilog2016), Asociación Mexicana de Logísitica y Cadena de Suministro A. C. (AML). https://www.researchgate.net/profile/Magdiel_Lara2/publication/320336233_Caract erizacion_de_modelo_de_negocio_en_el_marco_de_industria_40/links/59de8c68a6f dcca0d3204d75/Caracterizacion-de-modelo-de-negocio-en-el-marco-de-industria40.pdf

Rebolledo, E., Duque, C., López, L., \& Velasco, A. (2013). Perfil del sector manufacturero Colombiano Profile of Colombian manufacturing sector. Magazín Empresarial, 9(19), 49-61.

Restrepo-Morales, J. A., \& Vanegas-López, J. G. (2015). Internacionalización de las pymes: Análisis de recursos y capacidades internas mediante lógica difusa. $\begin{array}{llll}\text { Contaduria } y & \text { Administracion, 80(4), 836-863. }\end{array}$ https://doi.org/10.1016/j.cya.2015.07.008

Rojko, A. (2017). Industry 4.0 concept: Background and overview. International Journal of Interactive Mobile Technologies, 11(5), 77-90. https://doi.org/10.3991/ijim.v11i5.7072

Schuh, G., Anderl, R., Dumitrescu, R., Krüger, A., \& ten-Hompel, M. (2020). Industrie 4.0 maturity index. Managing the Digital transformation of companies. Update 2020. Acatech Study.

Schumacher, A., Erol, S., \& Sihn, W. (2016). A maturity model for assessing Industry 4.0 readiness and maturity of manufacturing enterprises. Procedia CIRP, 52, 161-166. https://doi.org/10.1016/j.procir.2016.07.040

Schwab, K. (2018). La Cuarta Revolución Industrial. Debate. 
Schwab, K. (ed.) (2019). The Global Competitiveness Report 2019. World http://www3.weforum.org/docs/WEF_TheGlobalCompetitivenessReport2019.pdf

Soldatos, J., Gusmeroli, S., Maló, P., \& Di Orio, G. (2016). Internet of things applications in future manufacturing. En O. Vermesan, \& P. Fries (eds.), Digitising Industry - Internet of Things connecting the physical, digital and virtual worlds (pp. 153-183). River

Publishers. https://www.researchgate.net/publication/305033020_Internet_of_Things_Applicati ons_in_Future_Manufacturing

Sommer, L. (2015). Industrial revolution - Industry 4.0: Are German manufacturing SMES the first victims of this revolution? Journal of Industrial Engineering and Management, 8(5), 1512-1532. https://doi.org/10.3926/jiem.1470

Vallejo-Zamudio, L. E. (2019). El Plan Nacional de Desarrollo 2018-2022: "Pacto por Colombia, pacto por la equidad". Apuntes del Cenes, 38(68). https://doi.org/10.19053/01203053.v38.n68.2019.9924

Vargas, R. (2008). Estadistica II. Programa Administración Pública Territorial. ESAP. http://www.esap.edu.co/portal/wp-content/uploads/2017/10/6-Estadsticaii.pdf

Vicepresidencia de Transformación Digital. (2019). Informe de la encuesta de transformación digital 2019. ANDI. http://www.andi.com.co/Uploads/ANALISIS ENCUESTA DE TRANSFORMACIÓN DIGITAL 2019 - ANDI.pdf

Vivares-Vergara, J. A. (2017). Modelo de madurez para valorar el sistema de producción y formular la estrategia de manufactura [tesis de doctorado, Universidad Nacional de Colombia]. Repositorio UN. https://repositorio.unal.edu.co/bitstream/handle/unal/62895/18617391.2017.pdf?se quence=1\&isAllowed=y

World Intellectual Property Organization [WIPO]. (2020). Global Innovation Index 2020. WIPO. https://www.wipo.int/global_innovation_index/es/2020/

Zorro-Galindo, E. (2019). Modelos de madurez digital en pymes - Caso de estudio de una pyme de telecomunicaciones de Colombia [tesis de grado, Universidad Nacional de Colombia]. Repositorio UN. https://repositorio.unal.edu.co/handle/unal/77419

Zurita-Heredia, A., \& Dini, M. (2021). Análisis de las políticas de apoyo a las pymes para enfrentar la pandemia de COVID-19 en América Latina. CEPAL. https://repositorio.cepal.org//handle/11362/46743 